\title{
Prevalência de hiperidrose em uma amostra populacional de Blumenau - SC, Brasil
}

\section{Prevalence of hyperhidrosis in the adult population of Blumenau-SC, Brazil}

\author{
Romero Fenili ${ }^{1}$ \\ Eder Deivis Fistarol ${ }^{3}$ \\ Lílian Mathias Delorenze ${ }^{5}$
}

\author{
Alexandre Roberto Demarchi \\ Mariana Matiello ${ }^{4}$
}

Resumo: FundAMENTOS: A hiperidrose é um distúrbio pouco conhecido, caracterizado pela produção excessiva de suor. Manifesta-se sob várias formas, afetando áreas como axilas, mãos, pés e face. Seus sintomas trazem prejuízos para o paciente, desde limitações no trabalho até constrangimento social e transtornos psicológicos.

OBjetivos: O objetivo deste trabalho é estimar a prevalência de hiperidrose na cidade de Blumenau.

Métodos: A pesquisa foi realizada entre 21 de agosto e $1^{2}$ de setembro de 2006 , nos terminais urbanos de Blumenau: SC, entrevistando-se 500 pessoas abordadas de forma aleatória.

REsultados: Dentre os entrevistados, 45 deles, que correspondem a 9\% do total, preencheram os requisitos mínimos para serem classificados como portadores de hiperidrose. A faixa etária mais acometida foi entre 18 e 30 anos (11,8\%). O número de portadores de hiperidrose do sexo masculino foi maior em números tanto absolutos quanto relativos. A característica mais prevalente foi frequência de hiperidrose ao menos uma vez por semana, encontrada em $91,11 \%$ dos afetados; a menos prevalente foi cessação de suor durante o sono, presente em $37,77 \%$.

CONCLUSÕES: Observa-se que a prevalência de hiperidrose em Blumenau é maior que a encontrada em outras pesquisas semelhantes, não evidenciando raridade na presente amostra.

Palavras-chave: Hiperidrose; Prevalência; Suor

\begin{abstract}
BACKGROUND: Hyperhidrosis is a little known disorder, characterized by excessive production of sweat. Hyperhidrosis may occur under several circumstances and appears in body parts like armpits, hands, feet, and face, consequently causing problems to the patient like job limitation, social embarrassment, and psychological damage.

Овјестілеs: Using these theoretical views as a starting point, the study aimed to evaluate the prevalence of hyperhidrosis in Blumenau.

Methodology: Utilizing interviews, the research was conducted with 500 subjects randomly approached, in the period ranging from August 21 to September 1, 2006.

Results: 45 respondents $(9 \%)$ matched the pre-requisites, being classified as hyperhidrosis patients. The age group most affected was from 18 to 30 years (11.8\%). Hyperhidrosis was more frequent among men both in absolute (24 men against 21 women) and relative numbers $(10.62 \%$ of men against $7.66 \%$ of women). The most prevalent characteristic was "at least one occurrence per week", in $91.11 \%$ of patients; the least prevalent occurrence was "cessation of sweat during sleep", in $37.77 \%$ of respondents. Conclusions: The data show that the prevalence of hyperhidrosis in Blumenau is higher when compared with other studies, thus indicating that it is not a rare phenomenon.

Keywords: Hyperhidrosis; Prevalence; Sweat
\end{abstract}

Recebido em 21.11.2008.

Aprovado pelo Conselho Consultivo e aceito para publicação em 08.06.09.

* Trabalho realizado no curso de Medicina da Universidade Regional de Blumenau (Furb) - Blumenau (SC), Brasil.

Conflito de interesse: Nenhum / Conflict of interest: None

Suporte financeiro: Nenhum / Financial funding: None

Doutor em Cirurgia do Tórax pela Universidade Autônoma de Barcelona (1996). Professor da Disciplina de Cirurgia Torácica da Fundação Universidade Regional de Blumenau (Furb) - Blumenau (SC), Brasil.

Acadêmico do $6^{\circ}$ ano de Medicina da Fundação Universidade Regional de Blumenau (Furb) - Blumenau (SC), Brasil.

Acadêmico do $6^{\circ}$ ano de Medicina da Fundação Universidade Regional de Blumenau (Furb) - Blumenau (SC), Brasil.

Acadêmica do $6^{\circ}$ ano de Medicina da Fundação Universidade Regional de Blumenau (Furb) - Blumenau (SC), Brasil.

Acadêmica do $6^{\circ}$ ano de Medicina da Fundação Universidade Regional de Blumenau (Furb) - Blumenau (SC), Brasil. 


\section{INTRODUÇÃO}

Hiperidrose é uma condição caracterizada pela produção excessiva de suor pelo corpo. Ela pode ser primária ou secundária. A hiperidrose primária não tem origem conhecida, mas ocorre devido à hiperatividade do sistema nervoso simpático. Ela apresenta-se localizada, geralmente simétrica, e pode afetar as axilas, as palmas das mãos, as plantas dos pés, a face e outras áreas. ${ }^{1}$

Evidências recentes sugerem que a hiperidrose primária tem um componente familiar, indicando base genética para essa condição. ${ }^{2}$

Por manifestar-se em áreas como axilas, mãos, pés e face, resulta em substancial prejuízo para o paciente, incluindo limitações no trabalho, na interação social, nas atividades físicas e no lazer, assim como transtornos psicológicos e de relacionamento. ${ }^{1}$

De acordo com uma pesquisa de qualidade de vida, os efeitos negativos da hiperidrose são comparáveis com os de condições tais como psoríase severa, insuficiência renal em estágio final, artrite reumatoide e esclerose múltipla. ${ }^{2}$

Dados epidemiológicos de prevalência de hiperidrose primária são escassos e insuficientes para uma estimativa precisa. Strutton e cols. citam, no seu artigo, um estudo realizado por Adar e col. entre jovens de Israel em 1977, em que encontraram uma prevalência de hiperidrose entre 0,6 e $1 \%{ }^{1}$

Moya e cols. descrevem em seu estudo a hiperidrose como uma condição que afeta mais de $1 \%$ da população. Já Han e cols. relatam uma incidência de $0,15-1 \% .4$

Strutton e cols., em uma recente pesquisa epidemiológica de 150.000 lares dos EUA, revelam uma prevalência de hiperidrose primária em $2,8 \%$ da população investigada. Mostram que a hiperidrose afeta igualmente ambos os sexos e que a prevalência é maior na faixa etária entre 25 e 64 anos. ${ }^{1}$

Lee e cols. descrevem a hiperidrose palmar como uma desordem funcional, comum em áreas subtropicais, com uma incidência estimada em 0,6-1\% em Israel e 1,6-3\% em Taiwan. ${ }^{5}$

De acordo com Alric e cols., a forma palmar é a mais debilitante, sendo observada em 0,5 a $1 \%$ da população. Os sintomas usualmente aparecem na puberdade, e 12 a 33\% dos pacientes apresentam história familiar. Essa condição é mais comum em mulheres (50 a 83\%). Estudos epidemiológicos têm demonstrado que não há evidências de predomínio em grupos étnicos ou localizações geográficas específicos. ${ }^{6}$

A prevalência de hiperidrose palmar foi observada em 4,59\% dos adolescentes da cidade de Fuzhou, na China, sem haver diferença significativa na prevalência entre os sexos. A história familiar de hiperidrose está presente em 15,3\% dos casos.
Conforme a pesquisa de Haider e col., a hiperidrose axilar afeta $51 \%$ dos pacientes, a plantar afeta $29 \%$, a palmar afeta $25 \%$ e a facial afeta $20 \%$. Nenhum estudo foi documentado sobre a história natural da doença com aumento da idade, mas, de acordo com a experiência clínica, a severidade do suor parece diminuir em pacientes com mais de 50 anos. ${ }^{8}$

A média de idade de início é 25 anos, mas depende principalmente da área afetada. A hiperidrose palmar e a hiperidrose axilar têm início precoce, em média aos 13 e 19 anos, respectivamente, sendo que aproximadamente $82 \%$ dos pacientes com a forma palmar relatam seu início na infância. ${ }^{1}$

A hiperidrose primária tem aparecido na infância, mas as pessoas afetadas, em geral, não procuram ajuda médica antes de se tornarem adultas. ${ }^{8}$

No Brasil não foi encontrada nenhuma pesquisa documentada, sendo um assunto pouco investigado.

Esta pesquisa visa a estimar a prevalência da hiperidrose na cidade de Blumenau, município localizado na região do Vale do Itajaí, no estado de Santa Catarina. É a terceira cidade mais populosa do estado, com 292.972 habitantes, e sua população é predominantemente de descendentes de europeus.'

\section{MATERIAL E MÉTODOS}

Este estudo é caracterizado como um estudo transversal e prospectivo.

\section{População e amostra}

Este trabalho foi realizado nos terminais urbanos do município de Blumenau, no período de 21 de agosto a $1^{\circ}$ de setembro de 2006 . Foram incluídas nesta pesquisa 500 pessoas de ambos os sexos e com idade igual ou superior a 18 anos.

A partir do estudo de Strutton e cols. (que estimou uma prevalência populacional de $2,8 \%$ ), o universo da pesquisa foi avaliado por meio de amostragem intencional, utilizando-se 5\% de erro amostral, o que resulta em uma amostra de no mínimo 400 indivíduos.

$\mathrm{O}$ trabalho foi realizado mediante aprovação do Comitê de Ética na Pesquisa com Seres Humanos da Universidade Regional de Blumenau, sob o número $061 / 06$.

\section{Procedimentos de coleta e análise de dados}

A coleta de dados foi feita através de entrevista com a aplicação de um questionário (Quadro 1), em que constavam os critérios diagnósticos para hiperidrose. Conforme pesquisa de Haider e col. ${ }^{8}$ para a definição de hiperidrose deve-se considerar a resposta positiva à primeira indagação e a presença de reação positiva para ao menos duas indagações subsequentes. 
QuADro 1: Questionário de pesquisa

Sexo: $M \quad$ F $\square \quad$ Idade: ( ) anos

Critérios diagnósticos para hiperidrose primária idiopática:

Apresenta suor excessivo, localizado e visível, por pelo menos seis meses de duração e sem causa aparente?

Sim $\square \quad$ Não

Além disso, apresenta mais duas das características listadas abaixo?

Suor bilateral e relativamente simétrico

Frequência de pelo menos 1 vez por semana

Prejuízo nas atividades diárias

Idade de início menor que 25 anos

História familiar positiva

Cessação do suor durante o sono

Piora em situações de estresse

Sem ou pouca interferência de temperatura

\section{Procedimentos e análise de dados}

Os dados foram analisados por meio de estatística descritiva e de testes de qui quadrado de independência para verificar associações entre os fatores pesquisados e a hiperidrose. Foram consideradas associações significativas para $\mathrm{P} \leq 0,05$.

\section{RESULTADOS}

Foram entrevistadas pessoas com idade igual ou superior a 18 anos, abordadas aleatoriamente nos terminais urbanos de Blumenau.

O número total de entrevistados foi de 500 pessoas, sendo 274 do sexo feminino e 226 do sexo masculino. Não houve diferença significante entre o número de entrevistados do sexo masculino e feminino com relação à hiperidrose $\left(\chi^{2}=0,98 ; \mathrm{gl}=1 ; \mathrm{P}=\right.$ $0,32)$. Portanto, não se pode afirmar que a hiperidrose dependa do sexo. Dentre os entrevistados, 45 (9\%) apresentaram os critérios diagnósticos para hiperidrose primária e 455 (91\%) não apresentaram tais critérios (Gráfico 1).

A amostra foi dividida por faixa etária em três classes: 18 a 30 anos, 31 a 59 anos e 60 a 83 anos.

Dentre as faixas etárias analisadas, encontrouse uma menor prevalência $(7,66 \%)$ de hiperidrose entre 31 a 59 anos, mesmo esta tendo o maior intervalo de idade e o maior número de entrevistados; a faixa etária entre 60 e 83 anos teve prevalência de $8,92 \%$; e a faixa etária que compreendia pessoas com idade entre 18 e 30 anos foi a que atingiu maior prevalência de hiperidrose, com $11,80 \%$. Entretanto, ao se realizar o teste de qui quadrado para verificar se houve uma associação entre a idade e a hiperidrose, tem-se que $\chi^{2}=2,04 ; \mathrm{gl}=2 ; \mathrm{P}=0,3614$. Portanto, não se pode afirmar que a hiperidrose dependa da idade (Gráfico 2).

A característica frequência de pelo menos uma vez por semana foi a que mais apareceu na pesquisa: 41 vezes; e a característica cessação do suor durante o sono foi a que menos apareceu: 17 vezes (Gráfico 3).

Quanto à relação entre sexos e características, evidenciou-se que somente as características bistória familiar positiva e piora em situações de estresse

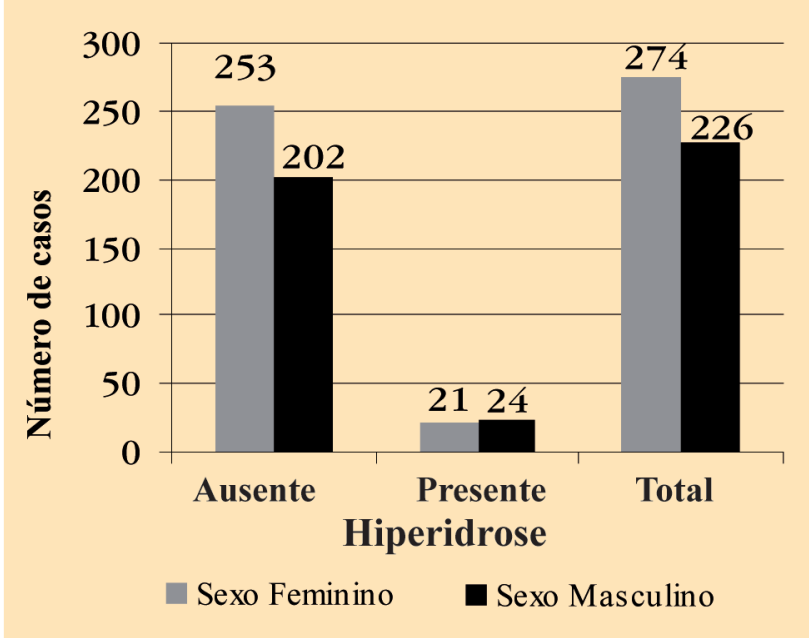

Gráfico 1: Prevalência comparativa entre os sexos 


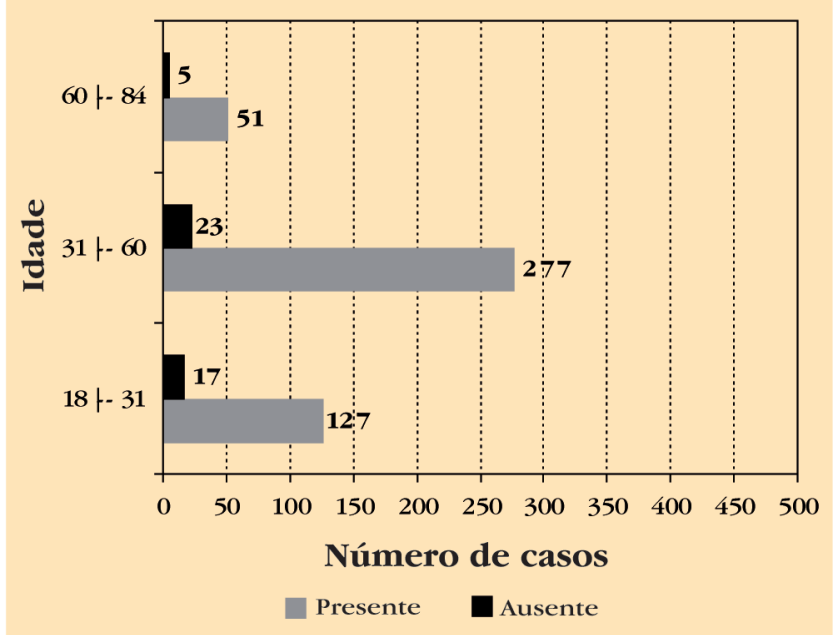

Gráfico 2: Prevalência de hiperidrose entre as faixas etárias

foram mais prevalentes no sexo feminino (Gráfico 4).

No geral, em relação às características levantadas aos entrevistados que sofrem de hiperidrose, revelou-se a seguinte ordem crescente de prevalência: cessação de suor durante o sono, história familiar positiva, idade de início menor que 25 anos, prejuízo nas atividades diárias, sem ou pouca interferência da temperatura, piora em situação de estresse, suor bilateral e relativamente simétrico e frequência de pelo menos uma vez por semana (Gráfico 5).

\section{DISCUSSÃO}

Em relação às pesquisas de Moya e cols., ${ }^{3}$ Han e cols. ${ }^{4}$ e Strutton e cols. ${ }^{1}$ que descreveram a hiperidrose como uma condição que afeta respectivamente mais de $1 \%$, de $0,15-1 \%$ e $2,8 \%$ da população, este estudo demonstrou números bem superiores, reve-

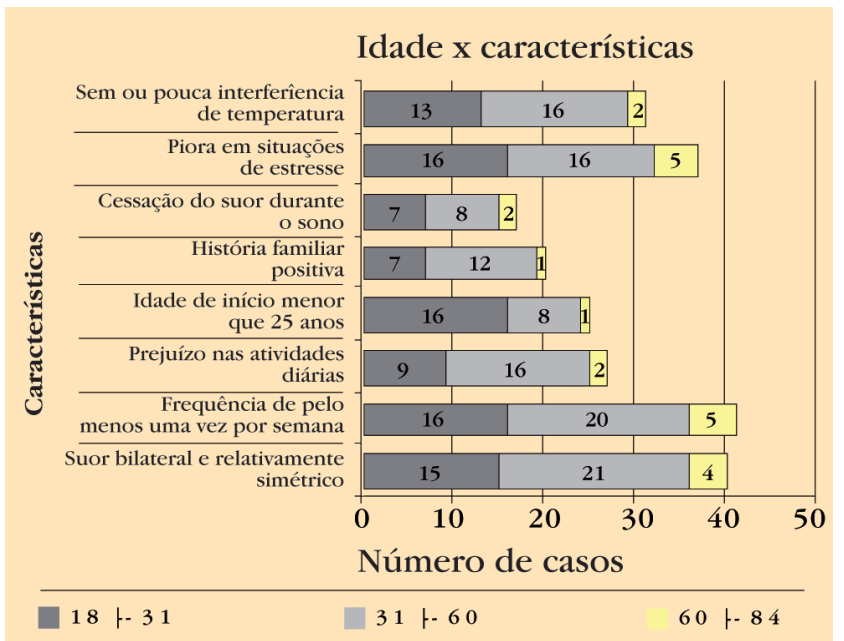

GráfICo 3: Frequência das características conforme faixas etárias

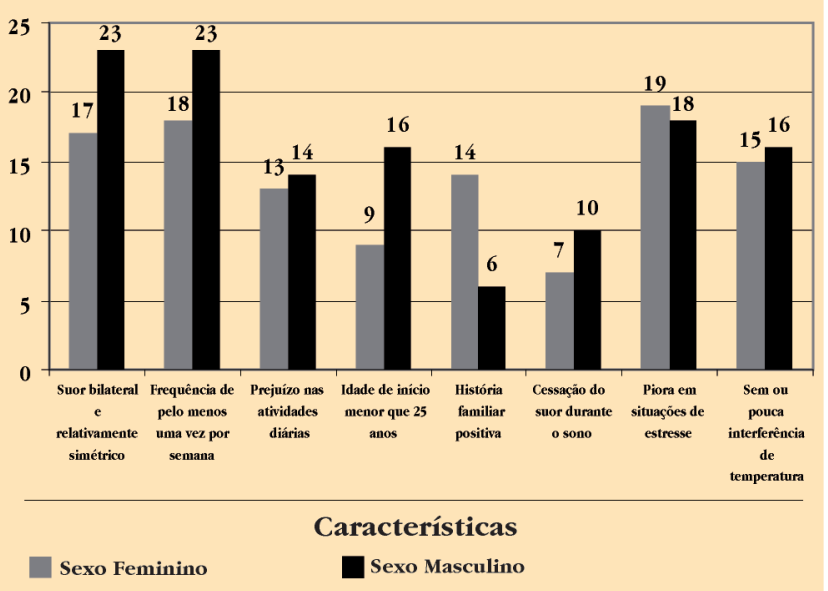

Gráfico 4: Frequência das características entre os sexos

lando que 9\% da população entrevistada de Blumenau são portadores desse distúrbio. Esse valor faz com que a hiperidrose deixe de ser um evento raro, ultrapassando índices próximos a 5\%.

Nesta pesquisa também evidenciou-se que há uma maior prevalência de afetados do sexo masculino (10,62\%) em relação ao sexo feminino (7,66\%), o que diverge um pouco da informação de Strutton e cols.,1 que declara que há paridade de homens e mulheres atingidos pela doença, e da de Tu e cols., ' que afirma não haver diferenças significativas de afetados entre os sexos.

De acordo com Tu e cols., $15,3 \%$ dos casos observados por eles em adolescentes na cidade de Fuzhou na China apresentavam história familiar positiva para hiperidrose. Já Alric e cols.6 descrevem que 12 a 33\% dos pacientes apresentam história familiar positiva.

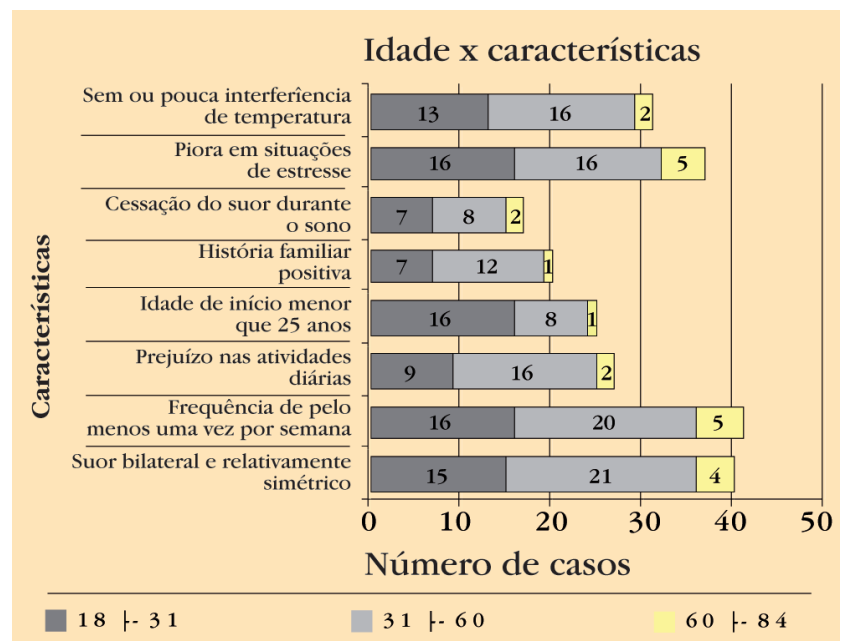

GráfICo 5: Prevalência das características entre os portadores de hiperidrose 
No presente trabalho, observou-se que cerca de $44,44 \%$ dos afetados possuem história familiar de hiperidrose.

Strutton e cols. ${ }^{1}$ demonstram em sua pesquisa que a prevalência de hiperidrose primária é maior em pessoas com idade entre 25 e 64 anos, ao passo que, neste estudo, foi a faixa etária entre 18 e 30 anos que apresentou maior prevalência $(11,80 \%)$, seguida das faixas etárias entre 60 e 83 anos $(8,92 \%)$ e entre 31 e 59 anos $(7,66 \%)$.

Conforme também refere o autor acima, a idade média de surgimento da hiperidrose é de 25 anos. $^{1}$

Apesar de o início dos sintomas de hiperidrose não ter sido questionado neste estudo, 25 entrevistados, que correspondem a $55,55 \%$ do total de porta- dores, relataram o surgimento desses sinais antes dos 25 anos.

\section{CONCLUSÕES}

A partir deste trabalho foi possível reconhecer uma estimativa da prevalência de hiperidrose de uma amostra da população adulta de Blumenau. O número de portadores desse distúrbio mostrou ser sensivelmente maior que o encontrado em outras pesquisas, revelando que a hiperidrose na cidade de Blumenau é um distúrbio mais comum do que se imaginava.

É importante a realização de novos estudos para esclarecer as reais prevalências desse distúrbio no país, pois, como revela o presente trabalho, a hiperidrose parece ser um evento muito mais frequente do que em princípio se divulga.

\section{AGRADECIMENTOS}

Dedicamos este trabalho a todos aqueles que, direta ou indiretamente, contribuíram para que fosse possível a sua realização, em especial ao Professor Carlos Efrain Stein pela sua assessoria estatística. $\mathrm{E}$, principalmente, àqueles que sofrem de hiperidrose, mas que, por falta de recursos ou até mesmo de informação, convivem com esse distúrbio e tentam levar suas vidas mais próximas do normal. 


\section{REFERÊNCIAS}

1. Strutton DR, Kowalski JW, Glaser DA, Stang PE. US prevalence of hyperhidrosis and impact on individuals with axillary hyperhidrosis: results from a national survey. J Am Acad Dermatol. 2004;51:241-8.

2. Hornberger J, Grimes K, Naumann M, Glaser DA, Lowe $\mathrm{NJ}$, Naver $\mathrm{H}$, et al. Recognition, diagnosis, and treatment of primary focal hyperhidrosis. J Am Acad Dermatol. 2004;51:274-86.

3. Moya J, Ramos R, Morera R, Villalonga R, Perna V, Macia I, et al. Thoracic sympathicolysis for primary hyperhidrosis: a review of 918 procedures. Surg Endosc. 2006;20:598-602.

4. Han PP, Gottfried ON, Kenny KJ, Dickman CA. Biportal thoracoscopic sympathectomy: surgical techniques and clinical results for the treatment of hyperhidrosis. Neurosurgery. 2002;50:306-11.

5. Lee KS, Chuang CL, Lin CL, Tsai LC, Hwang SL, Howng SL. Percutaneous CT-guided chemical thoracic sympathectomy for patients with palmar hyperhidrosis after transthoracic endoscopic sympathectomy. Surg Neurol. 2004;62:501-5.

6. Alric P, Branchereau P, Berthet JP, Léger P, Mary $H$, Mary-Ané C. Video-assisted thoracoscopic sympathectomy for palmar hyperhidrosis: results in 102 cases. Ann Vasc Surg. 2002; 16:708-13.
7. Tu YR, Li X, Lin M, Lai FC, Li YP, Chen JF, et al. Epidemiological survey of primary palmar hyperhidrosis in adolescent in Fuzhou of People's Republic of China. Eur J Cardiothorac Surg. 2007;31:737-9.

8. Haider A, Nowell S. Focal hyperhidrosis: diagnosis and management. CMAJ. 2005;172:69-75.

9. Instituto Brasileiro de Geografia e Estatística (IBGE) [homepage]. Contagem da população 2007 [acesso 11 Nov 2008]. Disponível em: www.ibge.gov.br/home/estatistica/populacao/ contagem2007/popmunic2007layoutTCU14112007.pdf

\footnotetext{
ENDEREÇO PARA CORRESPONDÊNCIA / MAILING ADDRESS:

Romero Fenili

Fundação Universidade Regional de Blumenau, Departamento de Medicina.

Rua Antonio da Veiga, 140 - Vila Nova

89010971 Blumenau SC

Tel./fax: 47 3321-0381 $473321-0381$

e-mail:fenili@furb.br
}

Como citar este artigo/How to cite this article: Fenili R, Demarchi AR, Fistarol ED, Matiello M, Delorenze LM. Prevalência de hiperidrose em uma amostra populacional de Blumenau - SC, Brasil. An Bras Dermatol. 2009;84(4):361-6. 